\title{
The safety, efficacy, and cost-effectiveness of gentamycin-collagen sponge in multicomponent prevention strategy of cardiac implantable electronic device infections - a single-center experience
}

\author{
Krzysztof Kaczmarek, Aleksander Strzelecki, Paweł Ptaszyński, Jerzy K Wranicz, Iwona Cygankiewicz
}

Department of Electrocardiology, Medical University of Lodz, Łódź, Poland

\section{Editorial}

by Baddour et al.

see p. 1055

Correspondence to: Krzysztof Kaczmarek, MD, $\mathrm{PhD}$,

Department of

Electrocardiology, Medical University of Lodz,

Pomorska 251, 92-213

Łódź, Poland

phone: +48 4220143 60,

e-mail: krzysztof.

kaczmarek@umed.lodz.pl

Copyright by the Author(s),

2021

Kardiol Pol. 2021;

79 (10): 1079-1085;

DOI: 10.33963/KP.a2021.0089

Received:

April 30, 2021

Revision accepted:

August 9, 2021

Published online:

August 9, 2021

\section{A B S TRACT}

Background: Cardiac implantable electronic device (CIED) infections are associated with significant morbidity, mortality, and increased healthcare expenses. Apart from standard systemic antibiotic therapy, locally acting agents are under investigation as a potential approach for the prevention of this complication.

Aims: The study aimed to summarize our experience with a gentamycin-collagen sponge (GCS) in a multi-component prevention strategy of cardiac implantable electronic device infection.

Methods: We retrospectively analyzed medical records of 312 consecutive patients who underwent CIED-related surgery and had at least a 6-month follow-up. All the individuals had GCS applied during surgery. An incidence of CIEDs-related infection in our group was compared to the risk level calculated according to the commonly used scores. Analysis of cost-effectiveness was also performed.

Results: Incidence of CIED-related infection, defined as a primary endpoint, occurred relatively rarely $(0.33 \%)$ as compared to the infection risk calculated according to commonly used scores Prevention of Arrythmia Device Infection Trial (PADIT) - $0.83 \%$; CIED-AI - $0.90 \%$ or Mittal score - $1.00 \% ; P<0.001$ - for all). We did not record any complications related to GCS. We analyzed the cost-effectiveness of our GCS-based approach, which appeared to be financially beneficial (number needed to treat 149-200; difference of CIED infection treatment cost and GCSs price was 5093-26525 \$).

Conclusions: We conclude that: (1) the use of GCS to reduce CIEDs infections is feasible and safe; (2) our multicomponent prevention strategy involving the GCS application seems to significantly reduce the rate of CIED infection, and it is cost-effective.

Key words: antibiotic prophylaxis, cardiac implantable electronic device, gentamycin-collagen sponge, infection

Kardiol Pol 2021; 79, 10: 1079-1085

\section{INTRODUCTION}

Cardiac implantable electronic devices (CIED), excluding subcutaneous implantable cardioverter-defibrillator (ICD), have a direct connection with the bloodstream and the cardiovascular system. As a result, a CIED infection (CDI) frequently leads to a life-threatening severe systemic infection (sepsis or/and infective endocarditis). The CDI rate 6-12 months after implantation was reported as $2.3 \%-3.4 \%[1,2]$ in retrospective studies, and $0.6-1.3 \%$ in prospective observational studies [3, 4], registries [5], the cross-over cluster Prevention of Arrhythmia Device Infection Trial (PADIT) [6], and randomized trials, World-wide Randomized Antibiotic Envelope Infection Prevention (WRAP-IT) [7]. CDIs are associated with a significant mor- 
The use of gentamycin-collagen sponge (GCS) to reduce cardiac implantable electronic devices infections (CDI) is feasible and safe. Our multi-component prevention strategy involving the GCS application seems to significantly reduce the rate of CDI and it is cost-effective.

tality rate, morbidity, and a significant financial healthcare burden [8-10].

Adequate prevention procedures should be implemented to avoid these catastrophic consequences. According to the European Heart Rhythm Association (EHRA) international consensus document [8], preventive measures are recommended to modify or eliminate several factors associated with CDI, present in pre-, peri- and postoperative periods. It is well-known that bacterial colonization and bacterial biofilm, ubiquitous in nature, are the most important cause of device-associated infection. As systemic antibiotic therapy has a limited impact on biofilm formation, antimicrobial agents that act locally or even on the surface of implants are thought to be promising [11]. In our center, we have implemented a multi-component prevention strategy involving systemic antibiotics and the administration of a local gentamycin-collagen sponge (GCS; garamycin). Gentamicin sulfate inhibits the synthesis of bacterial proteins and has a broad spectrum of antibacterial efficacy. Naturally structured collagen fibers of GCS activate blood coagulation, which prevents hematoma formation and, therefore, reduces the risk of bacterial colonization. GCS is successfully used to prevent a local infection of high-risk patients and in procedures in orthopedics and traumatology [12], cardiac surgery [13-15], and proctology [16]. There is only one single report in the Congress proceedings related to the prevention of CDI [17]. To the best of our knowledge, we present the first complete study on the application of GCS in the prevention of CDI.

\section{METHODS}

This retrospective observational study included all consecutive patients who underwent CIED implantation at the Electrocardiology Department of the Central University Hospital in Lodz in the third and fourth quarter of 2019. This analysis covered all classic devices implanted in the subclavicular region and connected to transvenous leads. Subcutaneous ICD, leadless pacemakers, and devices with epicardial leads were excluded. Data regarding demographics, laboratory tests, treatment, and clinical course including hospitalizations, procedural details, as well as out-patients clinic visits, were collected based on the hospital's electronic medical records. The follow-up was at least six months. In the case of missing follow-up records, we made phone calls to fill in this information. Special attention was paid to patient-related risk factors, including the history of device infection, chronic kidney disease, immunosuppression, chronic obstructive pulmonary disease (COPD), diabetes, chronic heart failure, New
York Heart Association class, skin disorders, malignancy, and antithrombotic medications. Then procedure-related details were collected: the type of procedure (de novo implantation, device replacement, revision, or upgrade), procedure duration, hematoma, need for early reintervention, temporary pacing, and antibiotic prophylaxis. Additionally, device-related details, such as the type of generator pocket (i.e., subclavian, abdominal pocket), type of lead (endocardial and epicardial), and the number of leads implanted were recorded. CDI was considered as a primary endpoint of our study; however, any type of complication related to CIED was carefully analyzed.

Infective complications were defined following the EHRA international consensus [8] and included: superficial incisional infection and CDI with 2 variants: pocket infection and CIED systemic infection/infective endocarditis. For precise pocket hematoma classification, we used the hematoma grading based on the recently published classification [18].

We have performed CIED implantation according to a locally established standard regarding pre-, intra-, and postoperative periods. Our preoperative management included an appropriate selection of patients eligible for CIED implantation, identification of risk factors associated with CDI and, if possible, their elimination (e.g., avoiding central venous catheters, discontinuation of antithrombotic therapy). We excluded patients with any current infection. Moreover, we inspected oral cavities and performed sanitation when required. Electrocoagulation used as a supportive measure for surgical hemostasis was considered as the standard of care. GCS with a dimension of $10 \times 10 \times 0.5 \mathrm{~cm}$, containing the aminoglycoside antibiotic gentamicin sulfate ( $200 \mathrm{mg}: 2 \mathrm{mg} / \mathrm{cm}^{2}$ ) and purified bovine collagens (type I - 95\% and type III - 5\%), was routinely put into the generator pocket. GCS provides a high local concentration of gentamicin (reached after 1-2 hours and maintained at this level for several days) with corresponding low serum levels [19, 20]. Based on relevant papers published previously, as well as nearly 20 years of experience at our center, in 2018 we implemented our recommendation for antibiotics prophylaxis, which was approved by the hospital team for the control and prevention of infections. We created a multi-component risk score system of CIED infections that defined the score 3 points as high risk (Table 1) [21]. Accordingly, in low CDI-risk patients, we recommended an intravenous administration of ceftriaxone $(2.0 \mathrm{~g})$ within $60-120$ minutes before the planned beginning of a procedure (vancomycin in case of allergy to cephalosporins or carriers of methicillin-resistant 
Table 1. Multicomponent CDI risk score system

\begin{tabular}{l|c}
\multicolumn{1}{c|}{ Factor } & Points \\
\hline $\begin{array}{l}\text { Early CIED-related surgical reintervention (within 90 } \\
\text { days) }\end{array}$ & 3 \\
Pocket hematoma & 3 \\
Temporary endocardial pacing & 3 \\
History of CDI & 3 \\
Infection or fever within 48 hours before CIED-related & \\
procedure & 3 \\
Hemodialysis & 3 \\
Chronic skin disorder & 3 \\
Immunosuppressive therapy & 2 \\
History of more than 3 CIED-related procedures & 2 \\
Index procedure duration of more than 2 hours & 1 \\
Diabetes mellitus & 1 \\
Chronic obstructive pulmonary disease & 1 \\
Chronic kidney disease (eGFR $<60$ ml/min/1.73 $\mathrm{m}^{2}$ ) & 1 \\
Many comorbidities defined as Charlson index of more & \\
than 3 & 1 \\
Congestive heart failure & \\
\hline Sum of the points &
\end{tabular}

Sum of the points

$<3$ - low-risk of CDI; 3 or more - high-risk of $C D I$

Abbreviations: $\mathrm{CDI}$, cardiac device infection; CIED, cardiac implantable electronic device; eGFR, estimated glomerular filtration rate

staphylococcus aureus). In the case of high-risk patients, we recommend prolonging antibiotic prophylaxis treatment up to 72 hours after the procedure. As described above, a fragment of GCS was obligatorily inserted into each generator pocket, which was subsequently closed with absorbable sutures. Thereafter, the remaining portion of GCS (approximately $1 / 5$ ) was placed below sutures on the subcutaneous tissue. A prolonged course of antibiotics was also indicated in the case of a pocket hematoma, fever, or symptoms of infection. If the patient's discharge was planned before the completion of antibiotic treatment, ambulatory oral therapy was prescribed (cefuroxime $0.5 \mathrm{~g}$ 2 times a day or in the case of allergy to cephalosporin clindamycin $0.6 \mathrm{~g} 3$ times a day).

Cost-effectiveness estimations were performed with the use of our own data and information published in the United Kingdom. Costs of CDI-related hospitalizations, CIEDs extractions, and implantations of new CIEDs systems, if necessary, were summed up as an approximation of expenses connected to CDI. Additionally, data from the United Kingdom were also used for cost-effectiveness calculations to give a broader perspective. These costs were recalculated in United States dollars (USD). The price of one GCS in our institution was approximately 79 USD, and in the data published in the United Kingdom - $£ 80$ [22], whereas the costs related to one patient with CDI in Great Britain were estimated at $£ 30958$ [3].

The study was performed in compliance with the Helsinki Declaration and with Good Clinical Practice standards and was approved by the local Bioethical Committee (No. RNN/175/20/KE). All the patients gave informed consent before the CIED-related procedure.

\section{Statistical analysis}

Statistical analysis was done using Statistica software (ver. 13, StatSoft Inc., Tulsa, OK, USA). Continuous variables are shown as mean (standard deviation [SD]) if normally distributed or as median (interquartile range [IQR]) otherwise. Categorical data are shown as numbers and frequencies. For $\mathrm{CDI}$ rate frequency and $95 \%$ confidence intervals were calculated $[6,23,24]$. A comparison between the expected CDI risk and the CDI rate was done in our study with the non-parametric one-sample Wilcoxon signed-rank test. Values of $P<0.05$ were deemed statistically significant.

For the cost-effectiveness analysis, the absolute risk reduction (ARR) was calculated according to common principles, as a difference between the estimated event rate and the experimental event rate. The number needed to treat (NNT) was estimated according to the equation: NNT $=1 /$ ARR [25]. The financial benefit of preventing one CDI was calculated as (NNT) $\times($ GCS price) and was compared to CDI-related costs.

\section{RESULTS}

We included 312 patients, who had undergone CIED implantations. We acquired all patients data mostly from the hospital or out-patient clinic data sets. In the case of 22 patients $(7.1 \%)$, the information was completed via phone calls. The study population consisted mainly of males (193 patients; 61.9\%). The mean age (SD) of our patients was 74.1 (10.8) years, and more than one-third (37.5\%) were elderly (over 80 years old). The majority of patients $(219 ; 70.2 \%)$ suffered from hypertension, almost half of the study population $(151 ; 48.4 \%)$ had chronic kidney disease with eGFR lower than $60 \mathrm{ml} / \mathrm{min}$, approximately one-third (34.9\%) had diabetes, and one in ten (30;9.6\%) patient had COPD. Atrial arrhythmias were diagnosed in 141 patients (45.2\%), and almost one-third $(88 ; 28.2 \%)$ had heart failure with reduced ejection fraction. Immunosuppression, the history of CIED infection or hemodialysis were rare. Fever just before implantation (within 48 hours) was not recorded for any individual. A vast majority $(249 ; 79.8 \%)$ of patients received antithrombotic therapy, most often antiplatelets (103; 33.0\%) and direct oral anticoagulants (96; 30.8\%). Detailed characteristics of the study population are shown in Table 2.

De novo implantations were performed in 219 (70.2\%) individuals, 57 (18.3\%) had device replacements, and $36(11.5 \%)$ patients underwent CIEDs revision or upgrade, of which $18(5.8 \%)$ had a transvenous lead extraction. A history of at least one surgery related to CIED, performed prior to the index procedure, was disclosed in 93 individuals (29.8\%). The duration of surgery longer than 2 hours was recorded in 32 patients (10.3\%). Procedural data are presented in detail in Table 3.

Periprocedural antibiotic therapy longer than recommended was applied in $53(17.0 \%)$ patients, and 8 (2.6\%) 
Table 2. Demographic and clinical characteristics of the study population

\begin{tabular}{|c|c|}
\hline Total number of patients & 312 \\
\hline Age, years & $74.1(10.8)$ \\
\hline Female/Male & $119(38.1 \%) / 193(61.9 \%)$ \\
\hline NYHA class & $2.0(1.0-4.0)$ \\
\hline Atrial fibrillation/Atrial flutter & $128(41.0 \%) / 13(4.2 \%)$ \\
\hline LVEF (\%) & $53.0(36.0-58.0)$ \\
\hline HFrEF - LVEF $<40 \%$ & $88(28.2 \%)$ \\
\hline Hypertension & $219(70.2 \%)$ \\
\hline eGFR, $\mathrm{ml} / \mathrm{min}$ & $62.5(46.9-78.1)$ \\
\hline$<30 \mathrm{ml} / \mathrm{min}$ & $25(8.0 \%)$ \\
\hline Hemodialysis & $4(1.3 \%)$ \\
\hline Prosthetic valve & $24(7.7 \%)$ \\
\hline Chronic obstructive pulmonary disease & $30(9.6 \%)$ \\
\hline Immunosuppressive drugs & $6(1.9 \%)$ \\
\hline Diabetes mellitus & $109(34.9 \%)$ \\
\hline History of previous CIED infection & $3(1.0 \%)$ \\
\hline Transvenous temporary pacing & $24(7.7 \%)$ \\
\hline Antithrombotic treatment & $249(79.8 \%)$ \\
\hline SAPT & $70(22.4 \%)$ \\
\hline DAPT & $33(10.6 \%)$ \\
\hline DOAC & $96(30.8 \%)$ \\
\hline VKA & $29(9.3 \%)$ \\
\hline Others & $31(9.9 \%)$ \\
\hline Previous CIED-related procedure & $93(29.8 \%)$ \\
\hline 1 & $61(19.6 \%)$ \\
\hline 2 & $25(8.0 \%)$ \\
\hline$\geq 3$ & $7(2.2 \%)$ \\
\hline
\end{tabular}

Abbreviations: CIED, cardiac implantable electronic device; DAPT, dual antiplatelet therapy; DOAC, direct oral anticoagulants; eGFR, estimated glomerular filtration rate; $\mathrm{HFrEF}$, heart failure with reduced ejection fraction; LVEF, left ventricular ejection fraction; NYHA, New York Heart Association; SAPT, single antiplatelet therapy; VKA, vitamin $\mathrm{K}$ antagonists

Table 3. The details of CIED-related procedures

\begin{tabular}{lc}
\hline No. of procedures & 312 \\
New pacemaker & $168(53.8 \%)$ \\
New ICD & $34(10.9 \%)$ \\
New CRT pacemaker/defibrillator & $17(5.4 \%)$ \\
Pacemaker generator replacement & $31(9.9 \%)$ \\
ICD generator replacement & $9(2.9 \%)$ \\
CRT generator replacement & $17(5.4 \%)$ \\
Revision/up-grade & $36(11.5 \%)$ \\
$\quad$ Transvenous lead extraction & $18(5.8 \%)$ \\
$\quad$ Early <3 months & $13(4.2 \%)$ \\
Duration of procedure & \\
$\quad \leq 2$ hours & $280(89.7 \%)$ \\
$>2$ hours & $32(10.3 \%)$ \\
Left-sided CIED system implantation & $306(98.1 \%)$ \\
Right-sided CIED system implantation & $6(1.9 \%)$ \\
Subcutaneous pocket & $312(100 \%)$ \\
Submuscular pocket & $0(0.0 \%)$ \\
\hline
\end{tabular}

Abbreviations: CRT, cardiac resynchronization therapy; ICD, implantable cardioverter-defibrillator; other — see Table 2

had an atypical antibacterial regimen administered, which consisted of different than suggested antibiotics and dosage. Temporary intravenous pacing $(16 ; 5.1 \%)$, respiratory or urinary tract infection $(11 ; 3.5 \%)$, early surgical reintervention related to CIED $(5 ; 1.6 \%)$, delay due to rescheduling of implantation $(5 ; 1.6 \%)$, and concomitant Lyme disease
Table 4. Risk of cardiac device infection and study outcomes

\begin{tabular}{lc} 
Mittal risk, \% & $1.00[1.00-3.40]^{\mathrm{a}}$ \\
CIED-AI risk, \% & $0.90[0.00-0.90]^{\mathrm{a}}$ \\
PADIT risk, \% & $0.83[0.46-1.06]^{\mathrm{a}}$ \\
Cardiac device infection & $1(0.33 \%)^{\mathrm{a}}$ \\
Superficial incisional infection & $3(0.99 \%)^{\mathrm{a}}$ \\
Death (causes) & $20(6.4 \%)$ \\
Stroke & $5(1.6 \%)$ \\
Malignancy & $5(1.6 \%)$ \\
Heart failure & $3(1.0 \%)$ \\
Electric storm & $1(0.3 \%)$ \\
Chronic kidney disease & $1(0.3 \%)$ \\
Unknown & $5(1.6 \%)$ \\
Lead dislocation & $8(2.6 \%)$ \\
Pocket hematoma & $4(1.3 \%)$ \\
\hline
\end{tabular}

Data are shown as median [IQR] and number

${ }^{a}$ Calculated for patients who stayed alive for more than 6 months $(n=302)$

$(4 ; 1.3 \%)$ were the most frequently reported reasons for prolonged and atypical antibiotic therapy.

During the in-hospital stay, we observed 4 cases of pocket hematomas (1.3\%). There were 2 patients with first-grade pocket hematoma and 2 patients with thirdgrade pocket hematoma requiring discontinuation of antithrombotic therapy with prolongation of the antibiotic course and hospitalization. Hematomas were completely absorbed without any sequelae.

During the follow-up of at least 6 months (median [IQR]: 343 [266-420] days), 20 patients (6.4\%) died; of these, 10 patients (3.2\%) died within half a year from CIED-related surgery. None of the deaths was related to $\mathrm{CDI}$ or sepsis (Table 4). Eventually, the vast majority of patients (303; 97.1\%) completed a 6-month follow-up alive; therefore, these individuals were taken for further analysis of infectious complications related to CIEDs surgery.

Cardiac electronic device infection was disclosed in only one case $(0.33 \% ; 95 \% \mathrm{Cl}, 0.01 \%-0.97 \%)$. It was an isolated pocket infection that was diagnosed 45 days after an upgrade from a dual-chamber ICD to a cardiac resynchronization therapy-defibrillator (CRT-D). The infection was diagnosed in a 68-year-old male with persistent left superior vena cava and a high risk of $C D I$, according to all analyzed scores (our score -3 points; calculated CDI risk from CIED-AI score 2.50\%, Mittal score - 3.40\% and PADIT score $-3.45 \%)[6,23,24]$. This patient underwent extraction of the whole CIED system, and following intravenous antibiotic therapy, had a new CRT-D system implanted at the right side of the thorax. Additionally, a superficial incisional infection was diagnosed in other 3 patients $(0.99 \%$; $95 \% \mathrm{Cl}, 0.0-2.1 \%)$. All of them made a full recovery with completely healed wounds as a result of ambulatory oral antibiotic therapy.

Weassessed the risk of CDI in the patients who completed 6 months' follow-up using 3 popular score systems: CIED-AI score [23], PADIT score [6], and Mittal score [24] (Figure 1). The study population's CDI mean risk was approximately $1 \%$ if estimated with PADIT score (median [IQR]: 0.83 [0.46- 
-1.06]\%), CIED-Al score (median [IQR]: 0.90 [0.0-0.90]\%), and Mittal score (median [IQR]: 1.00 [1.00-3.40]\%). The $C D I$ rate in our study was significantly lower than the calculated risks $(P<0.001-$ for any score compared to the study results).

The number needed to treat with our GCS-based strategy to prevent one CDI ranged from 149 to 200 , depending on which risk estimation was taken for calculations $(0.83 \%-1.00 \%)$. CDI-related costs in our region were analyzed in a group of 12 consecutive patients referred to our center due to infection caused by implanted devices. The expenses related to the treatment of these patients ranged from 7531 USD to 36928 USD (mean [SD]: 20893 [8961] USD). Furthermore, the costs that resulted from the application of GCS to avoid one CDI were estimated at $11791-15800$ USD, if based on our local data, or $£ 11940$ -16000 (16653-22315 USD) for the United Kingdom. Therefore, the difference between the costs related to $C D I$ and the costs of avoiding this complication could be estimated between 5093-9102 USD and £14958-19018 respectively (20862-26525 USD), in favor of the GCS-based strategy.

\section{DISCUSSION}

The main findings of our study were that: (1) gentamycin-collagen sponges can be safely used for CDI prevention, and such a strategy seems to be beneficial in terms of the low infection rate; (2) the use of gentamycin-collagen sponges seem to be cost-effective.

Although systemic antibiotic treatment is highly effective in the prevention of bacterial contamination (and CIEDs infection) [26,27], it is inefficient in the elimination of bacterial biofilm, therefore, locally acting antibiotics were thought to be an interesting additive option. An antibacterial multifilament mesh envelope (TYRX ${ }^{\mathrm{TM}}$, Medtronic, Plymouth, MN, USA) eluting minocycline and rifampin reduced the incidence of CIED infection in the WRAP-IT trial (40\% lower incidence of a major CIED infection than standard-of-care infection-prevention strategies alone) [7]. An experimental study, using an in-vitro biofilm system, demonstrated that the antibacterial envelope inhibited the ability of Staphylococcus aureus to form biofilms on mock CIEDs [28]. Like TYRX, GCS releases antibiotics locally, and its efficacy in the eradication of bacterial biofilm was proved in vitro (on hydroxyapatite surface) [19]. Therefore, a high local concentration of antibiotics (in the case of GCS - gentamicin) maintained for several days in the generator pocket may prolong the inhibition of bacterial colonization [20]. In our center, a few years ago the above-mentioned rationale led to multidisciplinary consultations, which resulted in the elaboration of a multi-component prevention strategy of CDI that included application GCS during each CIED implantation apart from standard systemic antibiotic prophylaxis. This approach was incorporated into the internal recommendations of our institution, which were approved by the hospital team for infection control and prevention.
In our retrospective study, we showed the safety and efficacy of our strategy that included GCS. No complication related to GCS was observed, and the low rate of CDI was recorded (only one case, $0.33 \%$ ). It would have been expected approximately 3 patients with CDI (c.a. $1 \%$ in PADIT score, CIED-AI score and Mittal score). Additionally, the risk of CDI infection in retrospective studies is generally significantly higher than in prospective trials (2.3\%-3.4\%) $[1,2]$, which forecasts up to $10 \mathrm{CDI}$ in our population. If such predictions are compared with our results, it seems that our strategy might have saved from 2 to 8 patients from CDI.

Nonetheless, it must be underlined that our approach was not only based on GCS but included more aggressive systemic antibiotic therapy (ceftriaxone iv at least twice - one dose before the procedure and one the day after) [8], and additionally, a specific surgical technique of separate pocket closure with absorbable sutures. A worldwide survey [29] showed that longer than recommended [8] periprocedural antibiotic therapy is commonly applied, which is mainly due to the threat of CDI, recognized as a devastating complication. The surgical technique that we use could potentially reduce the rate of pocket hematoma, whose occurrence is related to an even nine-fold increased risk of CDI [30]. The rate of hematoma in the study population was $1.3 \%$, which was lower than rates generally reported in previously published studies (3.2\%-9\%) [31-33]. However, it should be noticed that GCS applied to the pocket might activate blood coagulation and prevent the hematoma $[12,16]$. Therefore, it could be possible that the use of GCS and our specific surgical technique might be considered as efficient measures to reduce the rate of pocket hematoma. Another important factor influencing $\mathrm{CDI}$ is the need for early reintervention, as well as long complex TLE procedures, which are connected with the higher risk of hematoma and prolonged hospital stay [34]. In our group, $13 \mathrm{CIED}$ surgeries were performed less than 3 months after the index procedure. The most frequent reasons were lead dislocation (2.5\%) and "dry" perforations $(0.95 \%)$, which were also reported elsewhere [31, 33, 35].

Our CDI-prevention strategy with the use of GCS seems to be cost-effective. Depending on which risk score is used, the number needed to treat for saving one patient with CDI was 149 to 200 for GCS, which was similar (40-166) to the TYRx antibacterial envelope $[7,36]$. Moreover, the unit price of GCS is much lower than the competing envelope (79-110 USD vs. 895 USD) [22, 36]. Thus, the use of gentamycin sponges would remain still cost-effective even if only $0.3 \%$ of patients were spared from CDI. As CDI is connected with catastrophic consequences [37], the GCS-based CDI prevention strategy should be considered in patients with a high risk of infective complication. However, having in mind the relatively favorable cost-effectiveness of such an approach, using the GCS strategy might be useful in all patients undergoing CIEDs-related surgery.

The study had several limitations, among them the most important were a single-center location, retrospective 
analysis, including a medium-size study population, and relatively short follow-up. On the other hand, this study reflected real-world management. The lack of personal contact with the patients, which was substituted with phone teleconsultations, could be a potential reason for the under-detection of CIED-related infections. However, due to the COVID-19 pandemic, this was the only possible option in some cases. Additionally, cost-effectiveness calculations have the potential of imprecision due to different equipment which is used for removal of CIEDs systems implanted 12 months or longer before the extraction than those inserted earlier.

Based on our results the following conclusions can be drawn. The use of gentamycin-collagen sponges to reduce cardiac electronic device-related infections is feasible and safe. Our multi-component prevention strategy involving the application of GCS seems to significantly reduce the rate of CIED infection and to be cost-effective. A further prospective and randomized multicenter study is needed to confirm our findings.

\section{Article information}

Conflict of interest: None declared.

Open access: This article is available in open access under Creative Common Attribution-Non-Commercial-No Derivatives 4.0 International (CC BY-NC-ND 4.0) license, allowing to download articles and share them with others as long as they credit the authors and the publisher, but without permission to change them in any way or use them commercially. For commercial use, please contact the journal office at kardiologiapolska@ptkardio.pl.

How to cite: Kaczmarek K, Strzelecki A, Ptaszyński P, et al. The safety, efficacy, and cost-effectiveness of gentamycin-collagen sponge in multicomponent prevention strategy of cardiac implantable electronic device infections - a single-center experience. Kardiol Pol. 2021; 79(10): 1079-1085, doi: 10.33963/KP.a2021.0089.

\section{REFERENCES}

1. Clémenty N, Carion PL, Léotoing L, et al. Infections and associated costs following cardiovascular implantable electronic device implantations: a nationwide cohort study. Europace. 2018; 20(12): 1974-1980, doi: 10.1093/europace/eux387, indexed in Pubmed: 29672690.

2. Ludwig S, Theis C, Brown B, et al. Incidence and costs of cardiac device infections: retrospective analysis using German health claims data. J Comp Eff Res. 2018; 7(5): 483-492, doi: 10.2217/cer-2017-0080, indexed in Pubmed: 29132224.

3. Ahsan SY, Saberwal B, Lambiase PD, et al. A simple infection-control protocol to reduce serious cardiac device infections. Europace. 2014; 16(10): 1482-1489, doi: 10.1093/europace/euu126, indexed in Pubmed: 24919539.

4. Uslan DZ, Gleva MJ, Warren DK, et al. Cardiovascular implantable electronic device replacement infections and prevention: results from the REPLACE Registry. Pacing Clin Electrophysiol. 2012; 35(1): 81-87, doi: 10.1111/j.1540-8159.2011.03257.x, indexed in Pubmed: 22077194.

5. Biffi M, Ammendola E, Menardi E, et al. Real-life outcome of implantable cardioverter-defibrillator and cardiac resynchronization defibrillator replacement/upgrade in a contemporary population: observations from the multicentre DECODE registry. Europace. 2019; 21(10): 1527-1536, doi: 10.1093/europace/euz166, indexed in Pubmed: 31209482.

6. Krahn A, Longtin Y, Philippon F, et al. Prevention of arrhythmia device infection trial: the PADIT trial. J Am Coll Cardiol. 2018; 72(24): 3098-3109, doi: 10.1016/j.jacc.2018.09.068, indexed in Pubmed: 30545448.

7. Tarakji KG, Mittal S, Kennergren C, et al. WRAP-IT Investigators. Antibacterial envelope to prevent cardiac implantable device infection. N Engl J
Med. 2019; 380(20): 1895-1905, doi: 10.1056/NEJMoa1901111, indexed in Pubmed: 30883056.

8. Blomström-LundqvistC, Traykov V, Erba PA, et al.ESC Scientific Document Group. European Heart Rhythm Association (EHRA) international consensus document on how to prevent, diagnose, and treat cardiac implantable electronic device infections - endorsed by the Heart Rhythm Society (HRS), the Asia Pacific Heart Rhythm Society (APHRS), the Latin American Heart Rhythm Society (LAHRS), International Society for Cardiovascular Infectious Diseases (ISCVID) and the European Society of Clinical Microbiology and Infectious Diseases (ESCMID) in collaboration with the European Association for Cardio-Thoracic Surgery (EACTS). Europace. 2020; 22(4): 515-516, doi: 10.1093/europace/euz246, indexed in Pubmed: 31702000.

9. Barbar T, Patel R, Thomas G, et al. Strategies to prevent cardiac implantable electronic device infection. J Innov Card Rhythm Manag. 2020; 11(1): 3949-3956, doi: 10.19102/icrm.2020.110102, indexed in Pubmed: 32368364.

10. Greenspon AJ, Patel JD, Lau E, et al. 16-year trends in the infection burden for pacemakers and implantable cardioverter-defibrillators in the United States 1993 to 2008. J Am Coll Cardiol. 2011; 58(10): 1001-1006, doi: 10.1016/j.jacc.2011.04.033, indexed in Pubmed: 21867833.

11. Darouiche RO. Device-associated infections: a macroproblem that starts with microadherence. Clin Infect Dis. 2001; 33(9): 1567-1572, doi: 10.1086/323130, indexed in Pubmed: 11577378.

12. Knaepler H. Local application of gentamicin-containing collagen implant in the prophylaxis and treatment of surgical site infection in orthopaedic surgery. Int J Surg. 2012; 10(Suppl 1): S15-S20, doi: 10.1016/j. ijsu.2012.05.020, indexed in Pubmed: 22659311.

13. Friberg $O$, Svedjeholm $R$, Söderquist Bo, et al. Local gentamicin reduces sternal wound infections after cardiac surgery: a randomized controlled trial. Ann Thorac Surg. 2005; 79(1): 153-162, doi: 10.1016/j. athoracsur.2004.06.043, indexed in Pubmed: 15620935.

14. Kowalewski M, Pawliszak W, Zaborowska K, et al. Gentamicin-collagen sponge reduces the risk of sternal wound infections after heart surgery: meta-analysis. J Thorac Cardiovasc Surg. 2015; 149(6): 1631.e1-1640.e6, doi: 10.1016/j.jtcvs.2015.01.034, indexed in Pubmed: 25703409.

15. Urbanowicz T, Straburzyńska-Migaj E, Buczkowski P, et al. Novel method of infection prophylaxis in heart transplantation by retrosternal gentamycin sponge application. Transplant Proc. 2015; 47(6): 1954-1957, doi: 10.1016/j.transproceed.2015.02.026, indexed in Pubmed: 26293080.

16. Hu X, Qiu L, Long S, et al. Efficacy and safety of local gentamicin collagen implanting for preventing SSI following colorectal surgery: A systematic review and meta-analysis. Int J Clin Exp Med. 2016; 9(9): 17750-17760.

17. Futyma PR, Gluszczyk R, Ciapala K, et al. P1497. Zero device-related infections in 4285 patient-years of follow-up after cardiac implantable electronic device replacement combined with topic gentamicin-collagen sponge application. EP Europace. 2017; 19(Suppl 3): iii306, doi: 10.1093/ehjci/eux158.123.

18. DE Sensi F, Miracapillo G, Cresti A, et al. Pocket hematoma: a call for definition. Pacing Clin Electrophysiol. 2015; 38(8): 909-913, doi: 10.1111/pace.12665, indexed in Pubmed: 25974662.

19. Maczynska $B$, Secewicz A, Smutnicka $D$, et al. In vitro efficacy of gentamicin released from collagen sponge in eradication of bacterial biofilm preformed on hydroxyapatite surface. PLoS One. 2019; 14(6): e0217769, doi: 10.1371/journal.pone.0217769, indexed in Pubmed: 31163049.

20. Sohail MR, Esquer Garrigos Z, Elayi CS, et al. Preclinical evaluation of efficacy and pharmacokinetics of gentamicin containing extracellular-matrix envelope. Pacing Clin Electrophysiol. 2020; 43(3): 341-349, doi: 10.1111/pace.13888, indexed in Pubmed: 32067241.

21. Charlson ME, Pompei $\mathrm{P}$, Ales $\mathrm{KL}$, et al. A new method of classifying prognostic comorbidity in longitudinal studies: development and validation. J Chronic Dis. 1987; 40(5): 373-383, doi: 10.1016/0021-9681(87)90171-8, indexed in Pubmed: 3558716.

22. Joshi V, Vaja R, Richens D. Cost analysis of gentamicin-impregnated collagen sponges in preventing sternal wound infection post cardiac surgery. J Wound Care. 2016;25(1):22-25, doi: 10.12968/jowc.2016.25.1.22, indexed in Pubmed: 26762494.

23. Calderón-Parra J, Sánchez-Chica E, Asensio-Vegas Á, et al. Proposal for a novel score to determine the risk of cardiac implantable electronic device infection. Rev Esp Cardiol (Engl Ed). 2019; 72(10): 806-812, doi: 10.1016/j. rec.2018.09.003, indexed in Pubmed: 30340923. 
24. Mittal S, Shaw RE, Michel K, et al. Cardiac implantable electronic device infections: incidence, risk factors, and the effect of the AigisRx antibacterial envelope. Heart Rhythm. 2014; 11(4): 595-601, doi: 10.1016/j. hrthm.2013.12.013, indexed in Pubmed: 24333543.

25. Cook RJ, Sackett DL. The number needed to treat: a clinically useful measure of treatment effect. BMJ. 1995; 310(6977): 452-454, doi: 10.1136/bmj.310.6977.452, indexed in Pubmed: 7873954.

26. de Oliveira JC, Martinelli M, Nishioka SA, et al. Efficacy of antibiotic prophylaxis before the implantation of pacemakers and cardioverter-defibrillators: results of a large, prospective, randomized, double-blinded, placebo-controlled trial. Circ Arrhythm Electrophysiol. 2009; 2(1): 29-34, doi: 10.1161/CIRCEP.108.795906, indexed in Pubmed: 19808441.

27. Da Costa A, Kirkorian G, Cucherat M, et al. Antibiotic prophylaxis for permanent pacemaker implantation: a meta-analysis. Circulation. 1998; 97(18): 1796-1801, doi: 10.1161/01.cir.97.18.1796, indexed in Pubmed: 9603534.

28. Agostinho A, James G, Wazni O, et al. Inhibition of Staphylococcus aureus biofilms by a novel antibacterial envelope for use with implantable cardiac devices. Clin Transl Sci. 2009; 2(3): 193-198, doi: 10.1111/j.17528062.2009.00123.x, indexed in Pubmed: 20443892.

29. Traykov V, Bongiorni MG, Boriani G, et al. Clinical practice and implementation of guidelines for the prevention, diagnosis and management of cardiac implantable electronic device infections: results of a worldwide survey under the auspices of the European Heart Rhythm Association. Europace. 2019; 21(8): 1270-1279, doi: 10.1093/europace/euz137, indexed in Pubmed: 31209483.

30. Essebag V, Verma A, Healey JS, et al. BRUISE CONTROL Investigators. Clinically significant pocket hematoma increases long-term risk of device infection: BRUISE CONTROL INFECTION study. J Am Coll Cardiol. 2016; 67(11): 1300-1308, doi: 10.1016/j.jacc.2016.01.009, indexed in Pubmed: 26988951.
31. Pakarinen S, Oikarinen L, Toivonen L. Short-term implantation-related complications of cardiac rhythm management device therapy: a retrospective single-centre 1-year survey. Europace. 2010; 12(1): 103-108, doi: 10.1093/europace/eup361, indexed in Pubmed: 19914920

32. Kutinsky IB, Jarandilla R, Jewett $M$, et al. Risk of hematoma complications after device implant in the clopidogrel era. Circ Arrhythm Electrophysiol. 2010;3(4): 312-318, doi: 10.1161/CIRCEP.109.917625, indexed in Pubmed: 20558847.

33. Piątek $\measuredangle$, Polewczyk A, Kurzawski J, et al. Analysis of the incidence and causes of repeated surgical interventions in patients with early complications electrotherapy -1 center experience from the period 2012-2015 [in Polish]. Przegl Lek. 2016; 73(6): 378-381, indexed in Pubmed: 29668205.

34. Prutkin JM, Reynolds MR, Bao H, et al. Rates of and factors associated with infection in 200909 Medicare implantable cardioverter-defibrillator implants: results from the National Cardiovascular Data Registry. Circulation. 2014; 130(13): 1037-1043, doi: 10.1161/CIRCULATIONAHA.114.009081, indexed in Pubmed: 25081281.

35. Palmisano $\mathrm{P}$, Accogli $\mathrm{M}$, Zaccaria $\mathrm{M}$, et al. Rate, causes, and impact on patient outcome of implantable device complications requiring surgical revision: large population survey from two centres in Italy. Europace. 2013; 15(4): 531-540, doi: 10.1093/europace/eus337, indexed in Pubmed: 23407627.

36. Ellis $C$, Kolek M. Rising infection rate in cardiac electronic device implantation; the role of the AIGISRx ${ }^{\circledR}$ antibacterial envelope in prophylaxis. Comb Prod Ther. 2011; 1, doi: 10.1007/s13556-011-0003-6.

37. Nowosielecka D, Polewczyk A, Jacheć W, et al. Transesophageal echocardiography for the monitoring of transvenous lead extraction. Kardiol Pol. 2020; 78(12): 1206-1214, doi: 10.33963/KP.15651, indexed in Pubmed: 33078921 . 